\title{
Animating Molecular Machines
}

\section{Janet Iwasa}

\section{University of Utah, Salt Lake City, Utah, United States}

Over the past decade, there has been a significant increase in the use of three-dimensional (3D) animation to depict molecular processes. Much of this growth has been made possible by recent advances in imaging technologies and methodologies. We have now accumulated diverse datasets that describe molecular structures, dynamics, functions, interactions and localization with increasing clarity and confidence, allowing researchers to construct detailed hypotheses or mental models of molecular processes that synthesize these data. Although these hypotheses are increasingly three-dimensional and dynamic, the visualizations researchers create (most commonly seen as model figures) often fail to capture this complexity [1]. This gap between how we imagine molecular processes and the visualizations we create to depict them motivated me to learn how to use 3D animation software to create scientific visualizations.

As a molecular animator, I use software from the entertainment industry to create visually rich animations that depict a molecular process that accurately reflects a specific hypothesis. During this presentation, I will share a number of insights drawn from over a decade of experience creating molecular animations with numerous research collaborators on diverse molecular topics. I will describe the workflow my group members and I have developed for creating a molecular animation and share some observations we have made on how the animation process can help researchers refine and explore their hypotheses. I will also demonstrate how 3D animation can be particularly beneficial for understanding and describing dynamic and complex molecular machines [2] and large molecular assemblies within the context of the cell. Molecular animations have also made their way into the educational and public stages, and have proven to be useful for engaging diverse audiences. I will discuss the importance of broad scientific communication and describe various outreach projects we have undertaken. Finally, I will discuss past and current efforts towards building tools that will enable researchers to more easily construct their own molecular visualizations.

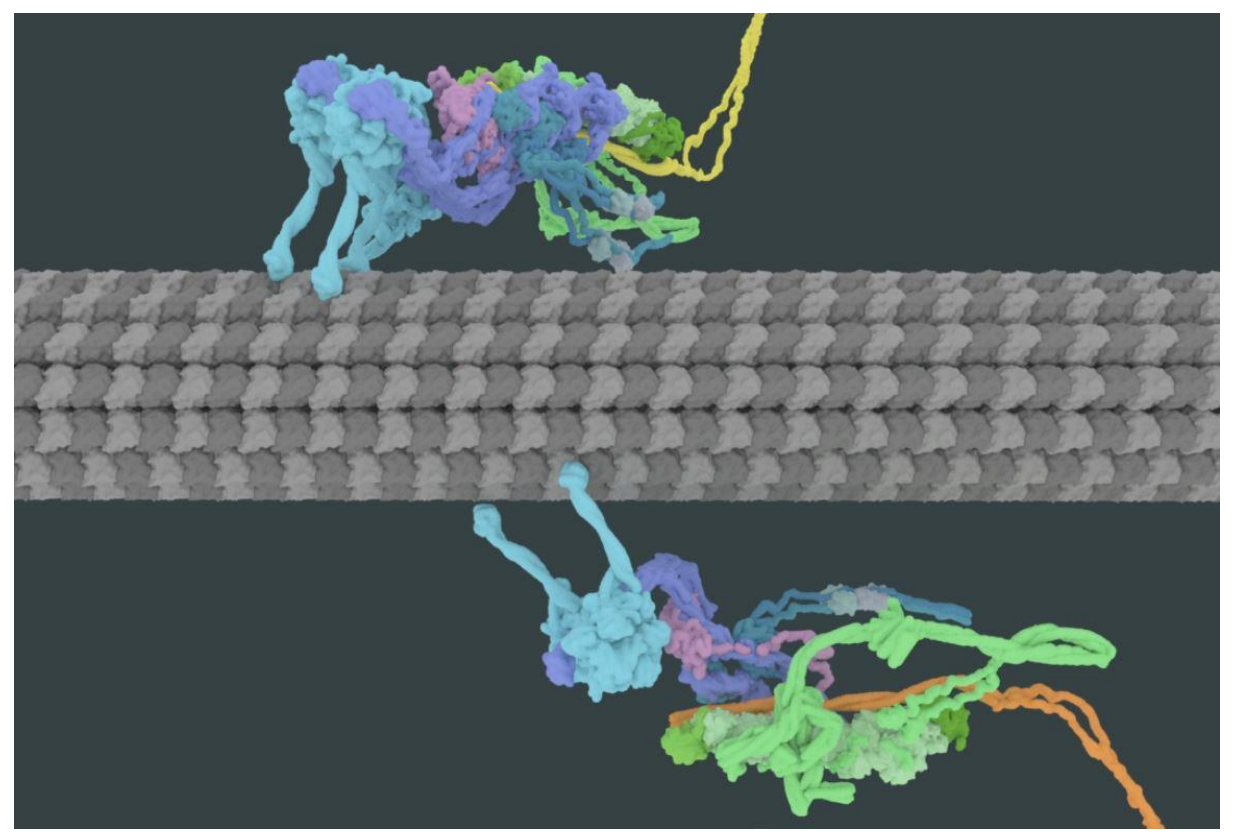


Figure 1. A still image from a 3D animation of the motor protein dynein, created in collaboration with Andrew Carter and Samara Reck-Peterson.

\section{References}

[1] Animating the model figure. Iwasa JH. Trends Cell Biol. 2010 Dec;20(12):699-704.

[2] Bringing macromolecular machinery to life using 3D animation. Iwasa JH. Curr Opin Struct Biol. 2015 Apr;31:84-8. 